\title{
Construct Optimal Binary Search Tree by Using Greedy Algorithm
}

Chun Shi ${ }^{1,}$, Ming Zhao ${ }^{1}$, Chunyu Li ${ }^{1, b}$, Chunlei Lin ${ }^{1}$ and Zhengjie Deng ${ }^{1}$

${ }^{1}$ School of Information Science \& Technology, Hainan Normal University, Haikou, Hainan 571127, China

ashichun@hainnu.edu.cn, lichunyu_hn@126.com

Keywords: Binary search tree; Greedy algorithm; Huffman tree; Efficiency; Complexity

\begin{abstract}
Focus on some constructions of binary tree, there are many methods to resolve this problem. With analyzes between binary search tree and Huffman tree, we introduce information retrieval issue and compare the Huffman tree with optimal binary search tree. And we further present a method that use greedy algorithm to construct binary search tree and use $\mathrm{C}++$ to realize method. Experimental provides some conclusions that greedy algorithm is more efficiency than dynamic programming algorithm.
\end{abstract}

\section{使用贪心算法构建最优二叉查找树方法}

\author{
石春 ${ }^{1, a}$, 赵明 ${ }^{1}$, 李春雨 ${ }^{1, b}$, 林春雷 ${ }^{1}$, 邓正杰 ${ }^{1}$ \\ 1. 海南师范大学 信息科学技术学院, 海南 海口 571127 \\ ashichun@hainnu.edu.cn, blichunyu_hn@126.com
}

摘要: 重点研究了二叉树的构成方法。在分析了二进制搜索树和哈夫曼树各自的特点, 针对 信息检索问题引入哈夫曼树与最优二叉搜索树。提出了一种采用贪心算法构造二进制搜索树, 并使用 $\mathrm{C}++$ 程序设计语言实现该方法。实验结果表明, 贪心算法比动态规划算法效率更高。

关键词: 二叉查找树;哈夫曼树;贪心算法;效率;复杂性

\section{1. 引言}

随着计算机技术的发展信息检索已经成为人们查找资源的重要方法，如何提高查找效率就成 为急需解决的问题。现在的信息检索技术分为两大类: 链式和树型结构, 但是树形存储结构 比线性存储结构更加灵活方便，这就是现在好多数据检索中经常用树形存储结构检索的原 因。目前构造树形结构进行检索的方法主要有：二叉排序树、平衡二叉树、红黑树、最佳二 叉排序树 [1-10], 这些树均只考虑结点在树中的深度, 不考虑结点的查找概率, 即：在所有 结点的查找概率相同的前提下构造二叉排序树。这些树是在所有结点的查找概率均相等的情 况下完成的。若在相同的概率下查找, 那么二叉排序树的平均比较次数是最少的。因为只考 虑结点的深度。如果所给各结点的查找概率不同，如何构造一棵最优的二叉查找树?

传统的方法都是用动态规划构造最优二叉查找树, 但是效率低下且容易产生内存溢出。基于 此原因，本文提出了一种使用贪心算法构建最优二叉查找树的方法。

\section{2. 问题分析}

事先给定 $n$ 维的向量 $\left(\mathrm{s}_{1}, \mathrm{~s}_{2}, \mathrm{~s}_{3}, \ldots . . . \mathrm{s}_{\mathrm{n}}\right) \mathrm{s}_{\mathrm{i}}$ 为向量中的元素 $1<=\mathrm{i}<=\mathrm{n}$ 且 $0<=\mathrm{s}_{\mathrm{i}}<=1$ 既 $\mathrm{s}_{\mathrm{i}}$ 为二叉树中查 找成功的概率。它具有如下性质:

(1) 如果 $S$ 为空集，则二叉树为一棵空树;

(2) 若 S 非空, 则存储在每个结点中的元素大于其左子树中任一结点所存储的元素, 于其右 子树中任一结点所存储的元素; 
（3）二叉查找树中的空指针对应形如 $\left(\mathrm{s}_{\mathrm{i}}, \mathrm{s}_{\mathrm{i}+1}\right)\left(i=0,1,2, \ldots n\right.$ 令 $\left.s_{0}=-\infty, s_{n+1}=+\infty\right)$ 的开区 间。

查找一个元素有两种可能:

（1）查找成功, 返回其在树中的位置 $\mathrm{s}=\mathrm{s}_{\mathrm{i}}$ （2）查找不成功, 返回空指针 $\mathrm{s} \in\left(\mathrm{s}_{\mathrm{i}}, \mathrm{s}_{\mathrm{i}+1}\right)$ 。 具有 $n$ 个结点的二叉树有个 $(n+1)$ 空指针。简单起见, 引入 $(n+1)$ 个结点 $e_{0} 、 e_{1} 、 \ldots . e_{n}$, 它 们代表个 $(\mathrm{n}+1)$ 空指针，其中 $\mathrm{e}_{0} \in\left(-\infty, \mathrm{s}_{1}\right), \mathrm{e}_{\mathrm{n}} \in\left(\mathrm{s}_{\mathrm{n}},+\infty\right), \mathrm{e}_{\mathrm{i}} \in\left(\mathrm{s}_{\mathrm{i}}, \mathrm{s}_{\mathrm{i}+1}\right) \quad(i=1,2, \ldots n-1)$ 。我们 称二叉查找树中 $s_{1} 、 s_{2} 、 \cdots$ 对应的结点为实结点, 即查找成功时的结点, 而称 $\mathrm{e}_{0} 、 \mathrm{e}_{1} 、 \ldots . \mathrm{e}_{\mathrm{n}}$ 为虚结点, 即: 查找失败时的结点 (空指针)。虚结点 $e_{j}$ 的查找概率为 $q_{j}(j=0,1,2, \cdots$, n) , 且 $\sum n_{i}=1 p_{i}+\sum n_{j}=0 q_{i}=1$ 。最优二叉查找树问题要求根据实、虚结点对应的关键字 和查找概率, 构造一棵平均比较次 ${ }^{[5]}$ 最少的二叉查找树。设在表示 $S=\left\{\mathrm{s}_{1} 、 \mathrm{~s}_{2}, \cdots, \mathrm{s}_{\mathrm{n}}\right\}$ 的二 叉查找树 $T$ 中, 元素 $s_{i}$ 的结点深度为 $c_{i}$ 查找概率为 $p_{i}$; 虚结点为 $\left\{e_{0}, e_{1} \cdots \cdots e_{a}\right\}$, 且 $e_{i}$ 的结点深度为 $d_{j}$, 查找概率为 $q_{j}$, 如果查找过程在实结点处结束, 需要进行比较的次数等于

实结点在二叉查找树中的深度加 1 ; 如果查找过程在虚结点处结束, 需要比较的次数等于 虚结点在二叉查找树中的深度。故二叉查找树的平均比较次数通常被表示为：

$\mathrm{C}=\sum \mathrm{n}_{\mathrm{i}}=1 \mathrm{p}_{\mathrm{i}}\left(1+\mathrm{c}_{\mathrm{i}}\right)+\sum \mathrm{n}_{\mathrm{j}}=0 \mathrm{q}_{\mathrm{j}} \mathrm{d}_{\mathrm{j}}$

直观地讲, 结点在二叉查找树中的深度越大, 需要比较的次数就越多。因此, 要构造一棵最 优二叉查找树, 应将查找概率高的结点靠近根结点。这种设计思想与哈夫曼树的构造思想相 似, 但最优二叉查找树所有结点的左右顺序 ( 这里指中序遍历的顺序)不能变化, 所以无法 像构造哈夫曼树的算法那样一味地把查找概率高的结点往上移。以 4 个字符的哈夫曼树和 3 个关键字的最优二叉查找树为例进行比较, 4 个字符 $\left\{S_{1} 、 S_{2} 、 S_{3} 、 S_{4}\right\}$, 对应的访问频率为 $\{0.1,0.4,0.35,0.15\}$, 其哈夫曼树如图 1-1 所示。3 个关键字 $\left\{s_{1} 、 s_{2} 、 s_{3}\right\}$, 对应的查找 概率为 $\{0.5,0.1,0.05\}$, 设置 4 个虚结点 $\left\{\mathrm{e}_{0} 、 \mathrm{e}_{1} 、 \mathrm{e}_{3}\right\}$, 对应的查找概率为 $\{0.15,0.1,0.05$, $0.05\}$ ，其最优二叉查找树如图 1-2 所示。

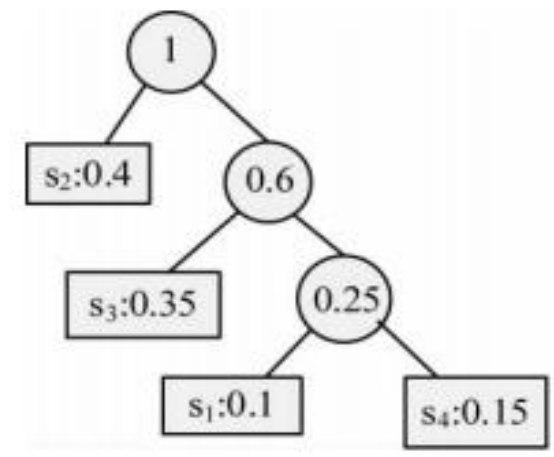

图 1-1 4 个字符的哈夫曼树

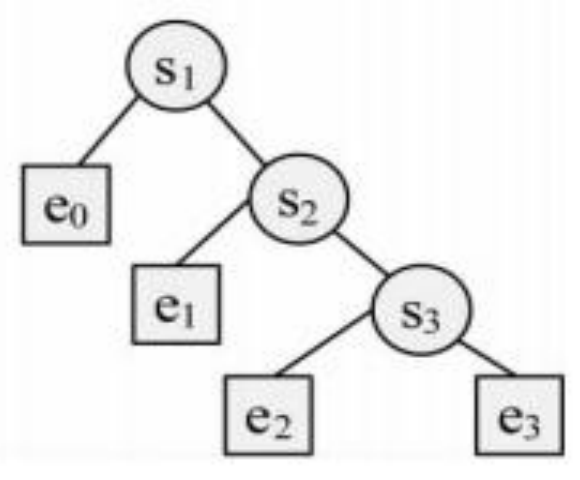

图 1-2 2 个关键字的最优二叉

图 1-1 所示的哈夫曼树编码的平均码长为: $0.4 \times 1+0.35 \times 2+0.1 \times 3+0.15 \times 3=1.85$, 图 2 所示 的最优二叉查找树的平均比较次数为: $0.5 \times 1+0.1 \times 2+0.05 \times 3+0.15 \times 1+0.1 \times 2+0.05 \times$ $3+0.05 \times 3=1.5$ 。认真对比哈夫曼树和最优二叉查找树, 不难发现：(1) 哈夫曼树和最优二叉 查找树都是二叉树; (2) ( $n+1)$ 个字符的哈夫曼树结点个数与 $n$ 个关键字组成的有序序列 $\mathrm{S}=\{、, \cdots$,$\} 的二叉查找树结点个数相同;（3）哈夫曼树要求字符编码的平均码长最$ 短, 最优二叉查找树要求平均比较次数最少, 平均码长和平均比较次数均由字符 (关键字) 在树中的深度直接决定, 所不同的是平均码长只考虑叶子结点在树中的深度和访问频率, 而 最优二叉查找树的平均比较次数既要考虑叶子结点在树中的深度和查找概率, 又要考虑中间 结点在树中的深度和查找概率; (4) 哈夫曼树中叶子结点对应待编码的字符, 中间结点为辅 助结点, 无实际含义; 最优二叉查找树中的叶子结点对应查找失败的虚结点, 中间结点对应 
序列 S 中的关键字; (5) 哈夫曼树对字符没有左右顺序的要求, 最优二叉查找树所有结点的 左右顺序 (这里指中序遍历的顺序) 不能变化。(6) 哈夫曼树中考虑的是访问频率和字符在树 中的深度，最优二叉查找树中考虑的是实结点及虚结点在树中的深度和查找概率。

\section{3. 贪心算法}

\section{1 . 设计思想}

用贪心算法构建最优二叉查找树算法的基本思想是将实虚结点分别看作是单结点树，把各结 点的权值概率按照 $\left\{e_{0} 、 s_{1} 、 e_{1}, \ldots, s_{n-1} 、 e_{n-1} 、 s n 、 e n\right.$ 的次序进行排列, 从中选出 $\left(q_{j-1}, p_{j}, q_{j}\right)$ 对应的最小结点 $\left(e_{j-1}, s_{j}, e_{j}\right)$, 然后从序列 $\left\{e_{0} 、 s_{1} 、 e_{1}, \ldots, s_{n-1} 、 e_{n-1} 、 s n 、 e n\right\}$ 中删除 $\left(\mathrm{e}_{\mathrm{j}-1}, \mathrm{~s}_{\mathrm{j}}, \mathrm{e}_{\mathrm{j}}\right)$, 生成以 为根结点, $\mathrm{e}_{\mathrm{j}-1}$ 为左子树, $e_{j}$ 为右子树的一棵新树, 将生成的新 树看成一个新的虚结点 $e^{\prime}$, 其权值为 $\mathrm{q}_{\mathrm{j}-1}+\mathrm{p}_{\mathrm{j}}+\mathrm{q}_{\mathrm{j}}$, 最后将虚结点插入到 $s_{j-1}$ 和 $s_{j+1}$ 之间, 以此类推, 直到把所有给出的结点合并成一棵树就可以了, 那么最后新构成的这棵树就被称 为最优二叉查找树。

\section{2. 算法步骤}

（1）构造数据结构, 选择链式的数据存储结构来插入, 删除节点。因为节点中有虚实节点故 使用两个单链表的数据结构来存储实虚结点. 其链表的语言描述为

Typedef struct node

$\{$ float data;

struct node $*$ next;

\} LNODE, * Link;

typedef struct \{

Link head, tail;

float sum;

int length;

\} * LinkList, List; / / 头尾结点

typedef struct \{

float min;

int foot;

\} * GetMin; / / 作为返回值的结构体 1, 用于去最小值部分

typedef struct \{

LinkList S1; / / 可表示实节点

LinkList S2；／/可表示虚节点

\}$*$ GetList; / / 作为返回值的结构体 2 , 用于主要部分作为返回值, 运用递推
（2）把实节点 $S=\left\{s_{1} 、 s_{2} 、 \cdots 、 s_{n}\right\}$, 分别构

（3）分别扫描两个单链表, 从 $\left(\mathrm{q}_{\mathrm{j}-1}+\mathrm{p}_{\mathrm{j}}+\mathrm{q}_{\mathrm{j}} \quad\right.$ ) 中选出最小的, 其对应的实虚结点分别 为 $\left(e_{j-1}, s_{j}, e_{j}\right)$ 且 $j=1, \ldots, n$ 组成以 $s_{j}$ 为根结点, $e_{j-1}$ 为左子树, $e_{j}$ 为右子树的一棵新树, 然后 删除掉实虚链表中相对应的结点, 把新树作为一个虚结点插入到虚链表中, 权值 为 $\mathrm{q}_{\mathrm{j}-1}+\mathrm{p}_{\mathrm{j}}+\mathrm{q}_{\mathrm{j}}$

(4) 重复 (3) 直到最后一个实结点和最后两个虚结点相加即完成所有操作。

\section{4. 算法实现与实例构造}

用 $\mathrm{C}++$ 程序设计语言实现求解最优二叉查找树的贪心算法, 设关键字个数为 $n$, 具体代码 如下: 
GetList CreatTree( GetList L, int num)

$\{$ int $\mathrm{i}$;

float min;

float a $[\mathrm{M}]$;

int foot; Link q, p;

GetMin MF;

MF $=($ GetMin $)$ malloc $($ sizeof $($ GetMin $)) ; / /$ 返回结构体类型 1

$\mathrm{p}=\mathrm{L}->\mathrm{S} 1->$ head $->$ next;

$\mathrm{q}=\mathrm{L}->\mathrm{S} 2->$ head $->$ next;

a $[0]=2$;

if ( num = =1) \{ printf( " 结束" ) ;

return L; \} / / 结束标志

else\{

for $(\mathrm{i}=1 ; \mathrm{i}<=$ num; $\mathrm{i}++)\{$

$\mathrm{a}[\mathrm{i}]=\mathrm{p}->$ data $+\mathrm{q}->$ data $+\mathrm{q}->$ next $->$ data;

$\mathrm{p}=\mathrm{p}->$ next;

$\mathrm{q}=\mathrm{q}->$ next; $\}$

MF $=$ Get_Min_number ( a, num, MF) ; // 返回结构体类型 1

$\min =M F->\min ; / /$ 数组中最小元素

foot $=M F->$ foot $/ /$ 数组中最小元素位置

$\operatorname{DelList1}(\mathrm{L}->\mathrm{S} 1, \mathrm{~L}->\mathrm{S} 2$, foot, a);

return L;// 返回结构体类型 2\} \}

该函数的流程图为下图所示

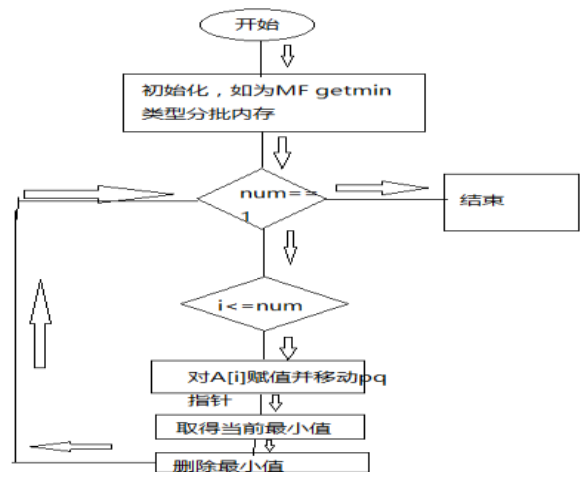

图 3-1 2 个关键字的最优二叉

\section{5. 结论}

本文提出了一种结合赫夫曼树的构造思想，构造了一种基于贪心算法的最优二叉查找树构建 方法, 该思想还是比较便捷的, 实验结果表明该算法的稳定性及其效率明显优于动态规划算 法.

\section{6. 致谢}

感谢国家自然科学基金项目（No. 61362016，61502127，61562022，61562023），海南省自然 科学基金项目（No. 20156225）和海南省高等学校科研项目（No. Hnky2016-17, Hnky2015-24） 支持。 


\section{Acknowledgement}

This research was financially supported by NSFC (No. 61362016, 61502127, 61562022, 61562023), NSF of Hainan Province (No.20156225) and scientific research project of Hainan Province (No.Hnky2016-17, Hnky2015-24).

\section{References}

[1] Yang Zhengan. Research and implementation of $\mathrm{C \#}$ based on two fork sort tree graphics display system [J]. Gansu science and technology, 2011, 27 (19): 27-28.

[2] Liang Daolei, Wang Qiufen. A greedy algorithm for constructing optimal two forks search tree [J]. computer applications and software, 2013, 30 (7): 57-61.

[3] Wang Wenxia. Construction of optimal binary search tree based on greedy algorithm [J]. Journal of Shanxi Normal University: Natural Science Edition, 2015 (1): 40-44.

[4] Wang Xingbo. Two fork tree deduction [J]. Computer engineering and applications, a fast algorithm to the serial number of the node intrinsic properties of 2011, 47 (9): 16-20.

[5] Wang Jue. A fast algorithm for the nearest common ancestor of adjacent nodes in the two fork tree [J]. Journal of Foshan University: Natural Science Edition, 2013, 31 (6): 55-58.

[6] Zhu Yu, Zhang Hongbin. The balance of the two fork tree selection adjustment algorithm [J]. Journal of University of science and China Institute, 2006, 23 (4): 527-533.

[7] Tang Faming, Wang Zhongdong, Chen Mianyun. A new algorithm of multi support vector machine with two cross trees [J]. Computer engineering and application, 2005, 41 (7): 24-26.

[8] Li Qiulin, Wang Jianjun, Gao Binbin. Two binary tree support vector multi classifier algorithm [J]. Journal of Southwestern University: Natural Science Edition, 2014 (7).

[9] Gao Qing, Jiang Fan. The red black tree algorithm and its application [J]. software guide, 2008 (9): 40-42.

[10] Wang Anhua, Li Bo. An optimal adaptive algorithm based on the two fork sort tree [J]. Journal of Yibin University, 2013, 13 (12): 77-80.

作者简介: 石 春 $(1977-)$, 男, 江西省鄱阳县, 副教授, 主要研究方向: 无线通信协议、 信息处理和软件工程, E-mail: byshichun@hainnu. edu. cn。 